\title{
Effect of high intensity focused ultrasound power level on device focal length
}

\begin{abstract}
In the past, several studies have been performed, investigating the thermal impact of high intensity focused ultrasound (HIFU) beams on real as well as artificial tissue samples. The goal behind such investigations is to develop HIFU as a reliable non-invasive thermal therapeutic modality for curing tumors. In order to ascertain the correct amount of thermal dosage delivered by the HIFU device or transducer it is important to develop a reliable method to determine the relationship between operating parameters like sonication time and power level on characteristics of the zone of ablation also called the lesion. While MRI thermometry and imaging and visual examination are attractive investigation techniques for determining lesion shape and size under variable conditions, investigating variation of focal distance or distance between the lesion center and transducer surface under variable conditions like power level, by such methods is difficult and has not been attempted. In order to corroborate and augment findings of MRI based HIFU studies, it is desirable to perform investigations using alternate methods. In this study, HIFU sonications were performed off the junction of thermocouples (embedded in a tissue mimicking phantom), in order to avoid thermocouple related artifacts. Analysis of temperature measurements revealed new and useful information regarding the effect of power level on focal length, an important performance characteristic. It was conclusively found that the HIFU focal length or beam location with respect to the transducer varied with power level, a rare and useful finding in HIFU studies.
\end{abstract}

Keywords: high intensity focused ultrasounds, tissue phantom, thermocouples, lesion, focal length, focal distance
Volume 5 Issue 3 - 2018

\author{
Subhashish Dasgupta \\ Mechanical Engineering, University of Cincinnati, USA
}

Correspondence: Subhashish Dasgupta, Mechanical Engineering, University of Cincinnati, Cincinnati, USA, Tel 7259818372,Email dasguptauc@gmail.com

Received: March 25, 2018| Published: May 08, 2018

\section{Introduction}

Extensive research has been performed in high intensity focused ultrasounds (HIFU) to develop an effective treatment method to destroy tumors or to retard their growth. The ability of guided HIFU to destroy or ablate tumors in selected tissue regions deep within the body while leaving intervening tissue undamaged is seen as an advantage of HIFU over other treatment procedures. Determination and quantification of the effect of the factors which influence HIFU ablation capabilities, is necessary, to decide the correct thermal dosage, to prevent destruction of the surrounding healthy cells due to over-dosage or to prevent recursion or incomplete damage of the tumor due to under-dosage.

A challenge encountered by HIFU researchers is development of an investigation technique to reliably characterize the thermal effect of HIFU transducer operating parameters in a real tissue sample or a tissue mimicking phantom. As recently as 2014, Solovchuk et al. ${ }^{1}$ performed HIFU studies on porcine muscle using MRI measurements together with numerical simulations. There was an acceptable agreement, especially at lower power levels, between MRI temperature measurements and simulations which accounted for nonlinearity. Also, lesion shapes within the tissue sample were examined by physically slicing the sample revealing distortion of shape and size at higher powers. In investigations performed by the present authors at an earlier date, ${ }^{2}$ MRI thermometry and imaging was performed to measure HIFU induced temperature rise in porcine liver samples and to measure lesion size at selected sonication times. A low pass filter was incorporated to overcome the effect of extraneous noise, affecting the scanning process. Comparing with numerical simulations it was found that temperature was measured with an $88 \%$ accuracy, similar to the accuracy levels achieved by Solovchuk et al. ${ }^{1}$ The present authors in their previous studies ${ }^{2}$ also found considerable distortion of lesion shape and size at higher power levels revealed by visual examination of lesions in sliced liver samples and MRI imaging.

A gap in the two investigations mentioned above as well as in other similar investigations, is that the possibility of variation of focal length of the transducer with increasing power level has not been conclusively established. The focal length is the distance of the HIFU beam from the transducer surface, a very useful parameter in accurate beam positioning during therapy or experimental studies. Visible examination of HIFU lesion images captured by MRI imaging or lesion photographs obtained from sliced tissue samples; do not provide conclusive evidence, of alteration of focal length with power level. These techniques reveal enlargement and distortion of lesions at higher powers, which is useful information. However, it is difficult to draw definitive conclusions regarding alteration of focal length with increasing power level. Hence in this study, a different method was used to investigate the possibility. Thermocouples were embedded in a tissue phantom and sonications were performed at increasing power levels at selected positions, off the thermocouple junctions to avoid artifacts (investigated by Dasgupta et al. ${ }^{4}$ ) The measured temperature was analyzed to draw conclusions about the possibility of focal length change at increased power level. 


\section{Methods}

\section{Experimental set up and sonicating procedure}

The possibility of alteration of HIFU transducer focal position at high power levels was investigated using a bench-top experimental set up. Figure 1a shows schematic of the set up. A HIFU transducer of focal length $10 \mathrm{~cm}$ was positioned atop a tissue mimicking phantom Figure 1, instrumented with thermocouples. The tissue mimicking phantom used was an agar-gel based phantom similar in acoustic and thermal properties as the one used by Menikou et al. ${ }^{3} \mathrm{~A}$ total of four thermocouples (T1, T2, T3, and T4) were used in the study. Thermocouples T1 and T2 were in one horizontal plane and $4 \mathrm{~mm}$ apart. Thermocouples T3 and T4, also $4 \mathrm{~mm}$ apart, were in a plane 4 $\mathrm{mm}$ above the plane of thermocouples $\mathrm{T} 1$ and T2. Figure $1 \mathrm{~b}$ shows 5 points at equal intervals between T1 and T2, where HIFU sonications were performed. Sonicating at the thermocouple junctions was avoided to prevent thermocouple artifacts from adulterating the measurements. Each sonication was performed for $30 \mathrm{~s}$ and transient temperature rise (when the transducer was powered on) and the transient temperature decay (when the transducer was powered off), were recorded. In this study, however, we are interested in temperature rise above the base or original temperature rise. At each sonicating point the sonication was repeated at higher powers. Initially a power level of $5 \mathrm{~W}$ was used, subsequently power levels of $17 \mathrm{~W}$ and $25 \mathrm{~W}$ were used.

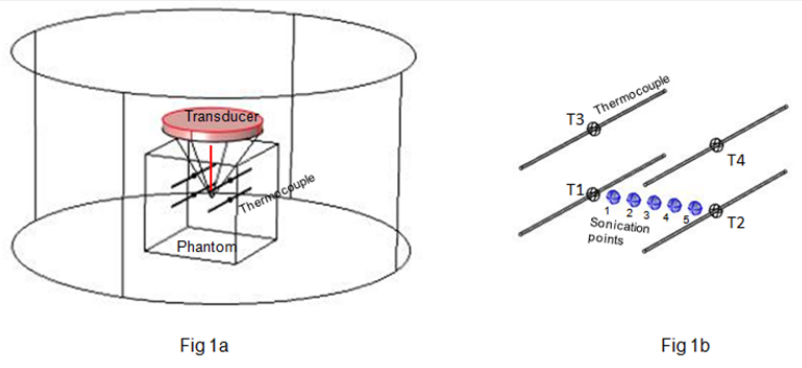

Figure I (A) Schematic of experimental set up showing HIFU transducer sonicating the phantom instrumented with thermocouples; (B) Thermocouple arrangement and sonicating points within thermocouple matrix.

In most HIFU investigations, degasified water is used to prevent ejection of gas bubbles during the negative pressure phase which could affect temperature rise and lesion formation (non-linearity issues). In this study ordinary water was used to accelerate the distortion of lesions at moderate powers. This is because of limitations with the transducer in elevating power levels to high values like $200 \mathrm{~W}$ as in the study by Solovchuk et al. ${ }^{1}$ Also, from the realistic point of view, organs under HIFU treatment are filled with blood which contains gas. Hence, our study could yield results that are closer to those obtained under in-vivo experiments involving anaesthetized animal specimens.

\section{Beam positioning}

Positioning the HIFU beam at the desired location is a challenge due to the extremely small diameter of the ellipsoid shaped beam (around a couple of $\mathrm{mm}$ ). A manual iterative positioning procedure was used in locating the beam on the selected locations. Initially the beam was approximately placed at T1, knowing the focal length of the transducer. Low power level sonications were performed around $\mathrm{T} 1$ at several locations at incremental distances from one another and temperature rise was recorded at each location. The sonicating point showing the maximum temperature rise at $\mathrm{T} 1$ was considered as location of the junction of thermocouple $\mathrm{T} 1$ and the positional coordinates (in three dimensions, $\mathrm{x}_{1}, \mathrm{y}_{1}, \mathrm{z}_{1}$ ) were recorded. Similarly, position of T2 was found and its coordinates recorded $\left(\mathrm{x}_{2}, \mathrm{y}_{2}, \mathrm{z}_{2}\right)$. Knowing the coordinates of T1 and T2, the distance between $\mathrm{T} 1$ and T2 was divided into 6 equal intervals. Hence, the 5 sonicating points $(1,2,3,4$, and 5$)$, indicated in Figure $1 \mathrm{~b}$, were determined.

\section{Results and discussion}

Figure $2 \mathrm{a}$ shows temperature rise after $30 \mathrm{~s}$, at sonicating points 1 to 5 (Figure 1b), recorded by thermocouples T1, T2, T3 and T4 at applied power level $5 \mathrm{~W}$. It is seen that as sonications are performed away from thermocouple $\mathrm{T} 1$, the temperature recorded by $\mathrm{T} 1$ drops and that recorded by $\mathrm{T} 2$ rises. Also, the corresponding temperatures recorded by T3, which is above T1, drops and T4, which is above T2, rises. All along the sonicating path, thermocouples $\mathrm{T} 1$ and $\mathrm{T} 2$ record a higher temperature rise than that recorded by $\mathrm{T} 3$ and $\mathrm{T} 4$. This is expected since the HIFU beam is on the plane of $\mathrm{T} 1$ and $\mathrm{T} 2 . \mathrm{T} 3$ and $\mathrm{T} 4$ are on a higher plane and measure lower temperatures than $\mathrm{T} 1$ and $\mathrm{T} 2$. However, Figure $2 \mathrm{~b}$ which shows temperature rise recorded at the 5 sonicating points at a higher power level of $17 \mathrm{~W}$, reveal that at most of the points, temperatures recorded by $\mathrm{T} 1$ and $\mathrm{T} 2$ are almost the same as that recorded by T3 and T4. In fact at some of the points the lower elevation thermocouples show a lower temperature rise than the higher elevation thermocouples. Figure 2c, which shows temperature measured at the points, at the highest power level used, $25 \mathrm{~W}$, shows an almost reverse trend to that seen in Figure 2a (lowest power level). It is seen that along the sonication points, temperature recorded by $\mathrm{T} 1$ and $\mathrm{T} 2$ are lower than those recorded by $\mathrm{T} 3$ and $\mathrm{T} 4$.
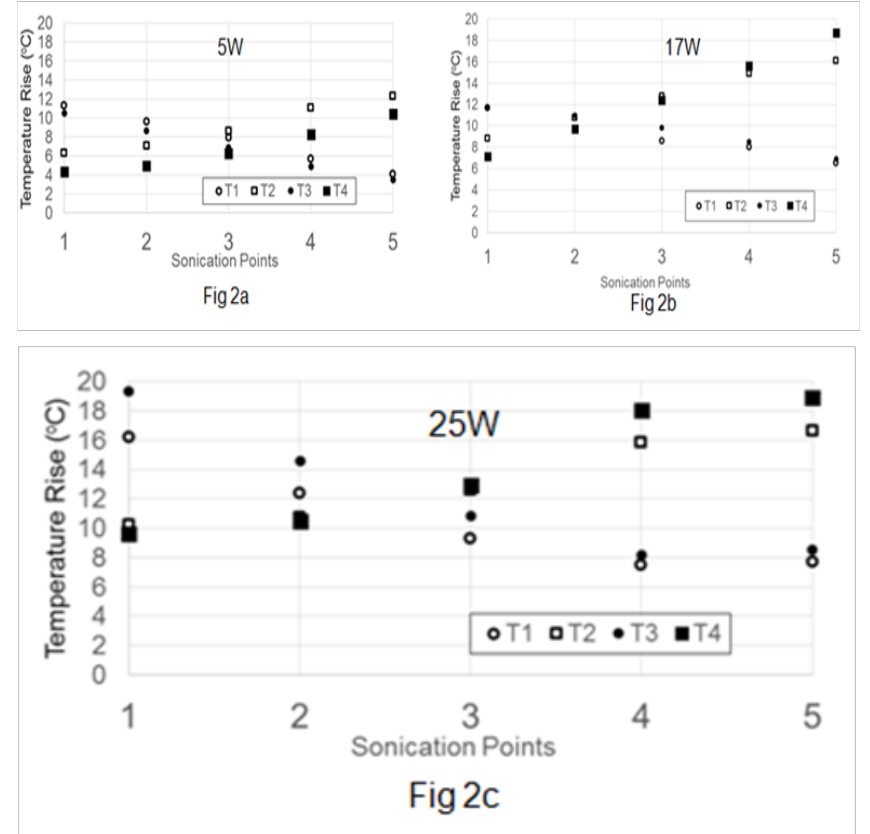

Figure 2 (A) Temperature rise at thermocouples, TI, T2, T3, T4, after $30 \mathrm{~s}$ sonication at selected sonication points, at power level 5W; (B \& C) Similar plots at power levels 17 and $25 \mathrm{~W}$ respectively.

From the above we may conclude that there is a definite effect of power level on the transducer focal distance. As power level increases from $5 \mathrm{~W}$ to $17 \mathrm{~W}$, the beam moves up from the plane of $\mathrm{T} 1-\mathrm{T} 2$ to a plane approximately midway between the planes of T1-T2 and T3-T4. 
This is evident from the temperatures measured by $\mathrm{T} 1$ and $\mathrm{T} 3$ being similar at most of the sonicating points (Figure 2b). Also temperatures measured by $\mathrm{T} 2$ and $\mathrm{T} 4$ are similar at most points at power level $17 \mathrm{~W}$. At the highest power level of $25 \mathrm{~W}$, temperature recorded by $\mathrm{T} 3$ is higher than that recorded by $\mathrm{T} 1$ and the temperature recorded by $\mathrm{T} 4$ is higher than that recorded by $\mathrm{T} 2$, at most points. This suggests that the beam has further moved up to a plane very close to the plane containing T3 and T4. Besides change in focal length, the possibility of the beam changing shape from ellipsoid to conical cannot be ruled out. This is explained further in the following paragraph discussing the results in the light of previous studies.

As mentioned earlier, MRI imaging and visual examination are useful methods to investigate effect of power level and other sonicating parameters like sonication duration, on lesion characteristics. Such methods reveal change of shape and size of lesions. Both Solovchuk and the present authors, Dasgupta et $\mathrm{al}^{2}{ }^{2}$ have shown that lesion characteristics substantially change with power level. Using MRI imaging both the studies show that lesion size increases with sonication power along the length and radius. In case of Solovchuk et al. ${ }^{1}$, lesions were created at power levels 140,160 and 200W, and found to elongate with increasing power. Further they showed that lesion shape changes from being ellipsoid to being conical at higher power. The base of the cone is at the transducer end. This could have also contributed to the fact that at higher power levels, in the present study, the higher elevation thermocouples (T3 and T4) measure a higher temperature rise than the lower elevation thermocouples (T1 and T2), the wide base of the conical beam being nearer T3 and T4 than to T1 and T2 During our previous study, ${ }^{2}$ apart from MRI imaging the ablated porcine liver was sliced and the lesions were visibly examined (study performed at Cincinnati Children's Hospital Medical Center). Figure 3a shows schematic of the MRI-HIFU experimental setup, where it is seen that a container holding the tissue sample is horizontally held in a Plexiglas box of degasified water and the transducer is focused on the tissue. Figure $3 \mathrm{~b}$ shows lesions formed at lower and higher power levels. It is seen that at higher applied power, the lesion departs from the typical ellipsoid shape to a conical shape, the possible effect of which has just been described. Also size of the lesion increases substantially. This study used degassed water and hence lesion size distortion occurred at much higher power levels of $70 \mathrm{~W}$.

However determining the change in focal length at high powers is difficult and at best can be qualitatively determined. MRI thermometry has been performed in both the studies ${ }^{1,2}$ and could possibly be useful in determining change in focal length with power. However MRI thermometry is not a well-established temperature measurement technique. Dasgupta et al. ${ }^{4}$ used a low pass filter to successfully overcome the issue of extraneous noise adulterating measurements. It can be anticipated that in future studies any stray noise source could render temperature measurements unreliable, without knowledge of the investigators. Solovchuk ${ }^{1}$ could measure temperature accurately at a lower power level. However at a higher power level a $15 \%$ measurement error was found.

Hence, while the MRI studies are useful in characterizing HIFU induced lesions, it will be desirable to augment the studies with an alternate method like thermocouple measurements. Temperature measured using thermocouples is not free of challenges. Direct sonication of thermocouple junctions could adulterate measurements with artifacts, studied in Dasgupta et al. ${ }^{4}$ Hence, in this study, sonications were performed off thermocouple junctions at selected locations, and the temperature thus measured was used in studying the effect of applied power level on focal length.

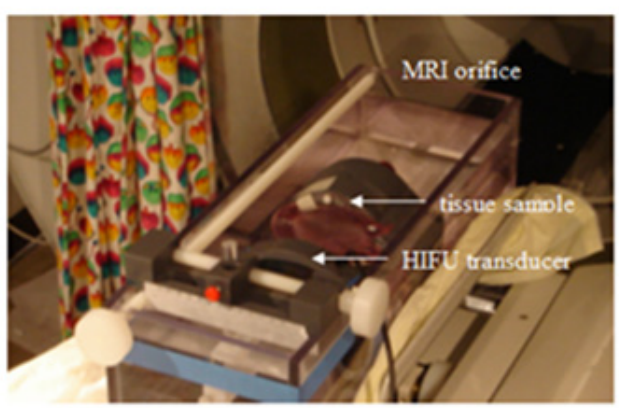

Courtesy: Cincinnati Chïdren's Hospital Medical Center (CCHMC)

\section{Fig 3a}

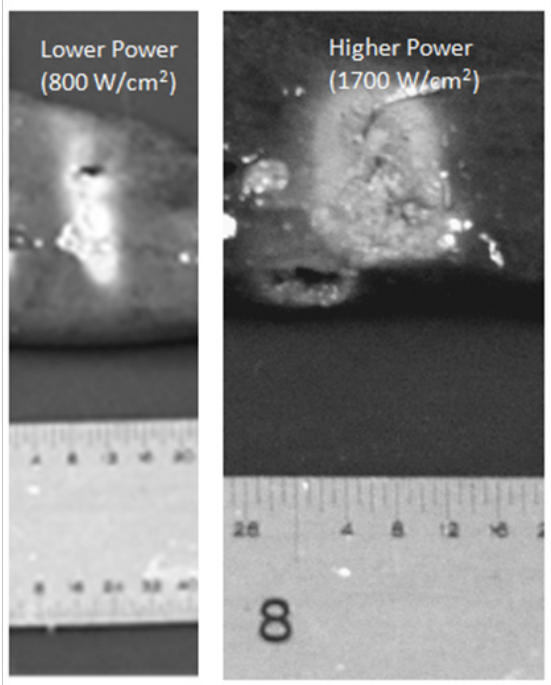

Fig $3 b$

Figure 3: (A) Experimental set up used to create and record HIFU induced lesions using MRI imaging. (B) Sonication of porcine liver at lower power (deep within tissue) and higher power (near tissue surface), reveals departure from ellipsoidal shape at higher power.

\section{Conclusion}

The intention of this study is not to suggest replacement of existing MRI or visual based studies, in investigating HIFU induced lesion characteristics under variable conditions. This is a study, which seeks to augment findings of existing studies. Several existing studies provide useful information regarding the change in lesion shape and size under the influence of parameters like power level and sonication duration. To the knowledge of the present authors there is no study that conclusively indicates alteration of HIFU focal distance with power level. Perhaps, in future, detailed studies can be performed quantifying the effect. It will be worthwhile accounting for such an alteration in future studies to obtain a complete picture in HIFU device characterization efforts. 


\section{Acknowledgements}

I acknowledge facility and support provided by Cincinnati Children's Hospital Medical Center (CCHMC) and University of Cincinnati, for a part of the studies.

\section{Conflict of interest}

The author declares there is no conflict of interest.

\section{References}

1. Solovchuk M, Hwang S, Chang H, et al. Temperature elevation by HIFU in ex-vivo porcine muscle: MRI measurement and simulation study. Med Phys. 2014;41(5):052903.
2. Dasgupta S, Wansapura J, Hariharan P, et al. HIFU lesion volume as a function of sonication time as determined by MRI, Histology and Computations. J Biomech Eng. 2010;132(8):081005.

3. Menikou G, Damianou C. Acoustic and thermal characterization of agar based phantoms used for evaluating focused ultrasound exposures. $J$ Ther Ultrasound. 2017;5:14.

4. Dasgupta S, Banerjee RK, Hariharan P, et al. Beam localization in HIFU temperature measurements using thermocouples, with application to cooling by large blood vessels. Ultrasonics. 2010;51(2):171-180. 